\title{
Metallographische Mitteilungen aus dem Institut für anorganische Chemie der Universität Göttingen. $\mathrm{XXXV}$. \\ Über Antimon-Kadmiumlegierungen.
}

\author{
Von \\ W. TREITSCHKE.
}

Mit 2 Figuren im Text.

Die älteren Angaben in der Literatur über Antimon-Kadmiumlegierungen beziehen sich lediglich darauf, dafs sich Antimon und Kadmium im flüssigen Zustand in allen Verhältnissen mischen ${ }^{1}$, sowie auf Gefrierpunktsbestimmungen zur Ermittelung des Molekulargewichtes $^{2}$, mithin auf ein sehr beschränktes Konzentrationsgebiet. Während ich mit der Untersuchung der Sb-Cd-Legierungen beschäft:gt war, erschien eine vorläufige Mitteilung von KURXakow und Konstantinow ${ }^{3}$ über diesen Gegenstand, deren Resultate im allgemeinen mit den meinigen übereinzustimmen scheinen.

Meine Untersuchung erfolgte anfangs in der Weise, dafs die Metalle in kleinen hessischen Tiegeln unter Kohlepulver zusammengeschmolzen wurden. Bei diesem Verfahren verdampfte aber eine wechselnde Menge Kadmium, so dafs die endgültigen Bestimmungen in schwer schmelzbaren Glasröhren in einer Kohlendioxydatmosphäre vorgenommen wurden, wobei die Konzentrationsänderung nicht mehr als $1 / 1000$ des Gehaltes an Kadmium betrug. Im übrigen kann betreffs des Versuchsverfahrens auf die Arbeit von GRUBE ${ }^{4}$ verwiesen werden.

${ }^{1}$ C. R. A lder Wright, Journ. Chem. Soe. Ind. 13 (1894), 1014-1020.

${ }^{2}$ C. Heycock und F. Nevilue, Proc. Chem. Soc. 6 (1890), 158. - Chem. News 62, 280. - Journ. Chem. Soc. 61 (1892), 888.

${ }^{3}$ Journ. russ. phys.-chem. Ges. 37 (1905), 580.

${ }^{4}$ G. Grube, Über Magnesium-Bleilegierungen, Z. anorg. Chem. 44, 117. 
Tabelle 1 enthält die Knicke und Haltepunkte bei der Abkühlung der Legierungen ohne Impfen, Tabelle 2 dieselben nach Impfung, welche kurz vor Beginn der Kristallisation vorgenommen wurde.

Das Zustandsdiagramm Fig. 1 bezieht sich auf die Gleichgewichte absolut stabiler Kristallarten und beschreibt die Vorgänge

\section{Tabelle 1.}

Kristallisation der stabilen Kristallart SbCd.

\begin{tabular}{|c|c|c|c|c|c|c|c|}
\hline \multicolumn{2}{|c|}{$\begin{array}{l}\text { Gehalt an Antimon } \\
\text { in }\end{array}$} & \multicolumn{2}{|c|}{$\begin{array}{l}\text { Beginn der } \\
\text { Kristallisation }\end{array}$} & \multicolumn{2}{|c|}{$\begin{array}{l}\text { Eutek tische } \\
\text { Kristallisation }\end{array}$} & \multicolumn{2}{|c|}{$\begin{array}{c}\text { Unwandlungs- } \\
\text { punkt }\end{array}$} \\
\hline $\begin{array}{l}\text { Gewichts- } \\
\text { prozenten }\end{array}$ & $\left|\begin{array}{c}\text { Atom- } \\
\text { prozenten }\end{array}\right|$ & Temp. & $\begin{array}{l}\text { Dauer } \\
\Delta z \frac{d z}{d t}\end{array}$ & Temp. & $\begin{array}{c}\text { Dauer } \\
\Delta z\end{array}$ & Temp. & $\begin{array}{l}\text { Dauer } \\
\Delta \boldsymbol{z} \frac{d z}{d t}\end{array}$ \\
\hline 100.0 & 100.0 & 632 & 130 & - & - & - & - \\
\hline 70.0 & 68.6 & 497 & & 462 & & - & - \\
\hline 68.16 & 66.7 & 493 & & 456 & 185 & - & 一 \\
\hline 65.0 & 63.5 & 479 & & 452 & 180 & - & - \\
\hline 60.0 & 58.4 & 455 & 130 & & & - & - \\
\hline 55.0 & 53.3 & 460 & 150 & & & - & - \\
\hline 51.64 & 50.0 & 456 & 160 & & & - & - \\
\hline 50.0 & 48.4 & 461 & 115 & & & & \\
\hline 45.0 & 43.4 & 453 & 60 & 296 & 60 & & \\
\hline 41.58 & 40.0 & 438 & 50 & 294 & 65 & & \\
\hline 40.0 & 38.4 & 437 & & 294 & 70 & & \\
\hline 30.0 & 28.6 & 386 & & 295 & 110 & & \\
\hline 26.25 & 25.0 & 368 & & 293 & 120 & & \\
\hline 20.0 & 19.0 & 340 & & 294 & 145 & 275 & 10 \\
\hline 15.0 & 14.2 & 325 & & 295 & & 280 & \\
\hline 10.0 & 9.4 & 295 & & 290 & 200 & 263 & 10 \\
\hline 7.5 & 7.1 & & & 293 & 225 & 264 & $\mathbf{5}$ \\
\hline 5.0 & 4.7 & 311 & & 297 & & 276 & \\
\hline 1.0 & 0.9 & 321 & & 291 & 10 & & \\
\hline Cd 100.0 & $\mathrm{Cd} 100.0$ & 323 & 90 & - & - & - & - \\
\hline
\end{tabular}

bei der Ablühlung derjenigen Reguli, welche bei Zimmertemperatur die für die Verbindung SbCd charakteristischen langen Nadeln enthalten.

Bei den Schmelzen zwischen 30 und $8 \%$ Antimon scheidet sich bei primärer Kristallisation die Verbindung SbCd immer spontan aus. Man erhält also bei spontaner Kristallisation und nach dem Impfen dieselben Punkte auf den Abkühlungskurven und auch Legierungen von derselben Struktur. 
Tabelle 2.

Kristallisation der nicht stabilen Kristallart $\mathrm{Sb}_{2} \mathrm{Cd}_{\mathbf{3}}$.

\begin{tabular}{|c|c|c|c|c|c|c|c|}
\hline \multicolumn{2}{|c|}{$\begin{array}{c}\text { Gehalt an Antimon } \\
\text { in }\end{array}$} & \multicolumn{2}{|c|}{$\begin{array}{l}\text { Beginn der } \\
\text { Kristallisation }\end{array}$} & \multicolumn{2}{|c|}{$\begin{array}{l}\text { Eutektische } \\
\text { Kristallisation }\end{array}$} & \multicolumn{2}{|c|}{ Umwandlung } \\
\hline $\begin{array}{l}\text { Gewichts- } \\
\text { prozenten }\end{array}$ & Atom- & Temp. & $\begin{array}{l}\text { Uaner } \\
\Delta z \frac{d z}{d t}\end{array}$ & Temp. & $\begin{array}{c}\text { Dauer } \\
\Delta x\end{array}$ & Temp. & $\begin{array}{l}\text { Höhe des } \\
\text { Temperatur- } \\
\text { sprungs }\end{array}$ \\
\hline 90.0 & 89.4 & 588 & & 408 & & 225 & 4 \\
\hline 80.0 & 78.9 & 548 & & 409 & & 237 & 14 \\
\hline 70.0 & 68.6 & 499 & & 407 & & 246 & 29 \\
\hline 68.16 & 66.7 & 486 & & 408 & 155 & 349 & 33 \\
\hline 65.0 & 63.5 & 470 & & 407 & 190 & 274 & 8 \\
\hline 60.0 & 58.4 & 440 & & 411 & 210 & 405 & 40 \\
\hline 55.0 & 53.3 & 411 & $125 ?$ & & & 411 & 45 \\
\hline 51.64 & 50.0 & 415 & 130 & & & 410 & 41 \\
\hline 48.0 & 46.4 & 420 & & & & 381 & 12 \\
\hline 45.0 & 43.4 & 424 & 130 & & & & Dauer $\Delta z \frac{d z}{d t}$ \\
\hline 41.58 & 40.0 & 419 & 130 & & & 304 & $10 \quad a t$ \\
\hline 40.0 & 38.4 & 414 & & 293 & 5 & 230 & 15 \\
\hline 35.0 & 33.5 & 398 & & 290 & 50 & 238 & 10 \\
\hline
\end{tabular}

Die Schmelzen von 30-70\% Sb mulsten geimptt werden, damit sich die Verbindung SbCd aus denselben ausschied. Geimpft wurde jede Legierung mit ihrem eigenen Pulver, von dem kurz vor der Kristallisation etwa $1 / 20 \mathrm{~g}$ auf $30 \mathrm{~g}$ der geschmolzenen Legierung geschüttet wurde.

Die Gründe, welche für die Formel SbCd der eben erwähnten in langen Nadeln kristallisierenden stabilen Verbindung sprechen, sind folgende: Lrstens wird die Zeitdauer der eutektischen Kristallisation $\left(292^{\circ}\right)$ bei etwa $51 \%$ Antimon Null, während dieser Haltepunkt bei der Legierung mit 51.64\% Antimon nicht mehr vorhanden ist. Zweitens hat die Zeitdauer der primären Kristallisation (auf der Horizontalen $p q$ aufgetragen) zwischen 60 und $40 \% \mathrm{Sb}$ ihren grölsten Wert bei ca. $\$ 2 \% \mathrm{Sb}$. Das der Verbindung SbCd entsprechende Maximum auf der Kurve der primären Kristallisation muls nach den Bestimmungen der Temperatur des Beginnes der Kristallisation zwischen 50 und $55 \%$ Sb liegen. Eine genaue Bestimmung der Konzentration des Maximums wäre nur möglich, wenn die Genauigkeit der Temperaturbestimmung bis auf $1 / 10^{0}$ gesteigert würde. Die Abkühlungskurve der Legierung, welche der Zusammensetzung der Verbindung SbCd entspricht, hat die Form der Ab- 
kühlungskurve eines chemisch homogenen Stoffes. Aber fast dieselbe Form haben auch die Abkühlungskurven von $55-60 \%$ Antimon. Während bei $50 \%$ Antimon die Temperatur bis zum Ende der Kristallisation um $4^{\circ}$ fällt, sinkt sie bei $51.6 \%$ um $5^{0}$, bei $55^{\circ} \%$ um $6^{\circ}$ und bei $60 \%$ um $8^{\circ}$. Auf Grund der Abkühlungskurven mülste man hier zwischen 52 und $60 \% \mathrm{Sb}$ eine Reihe von

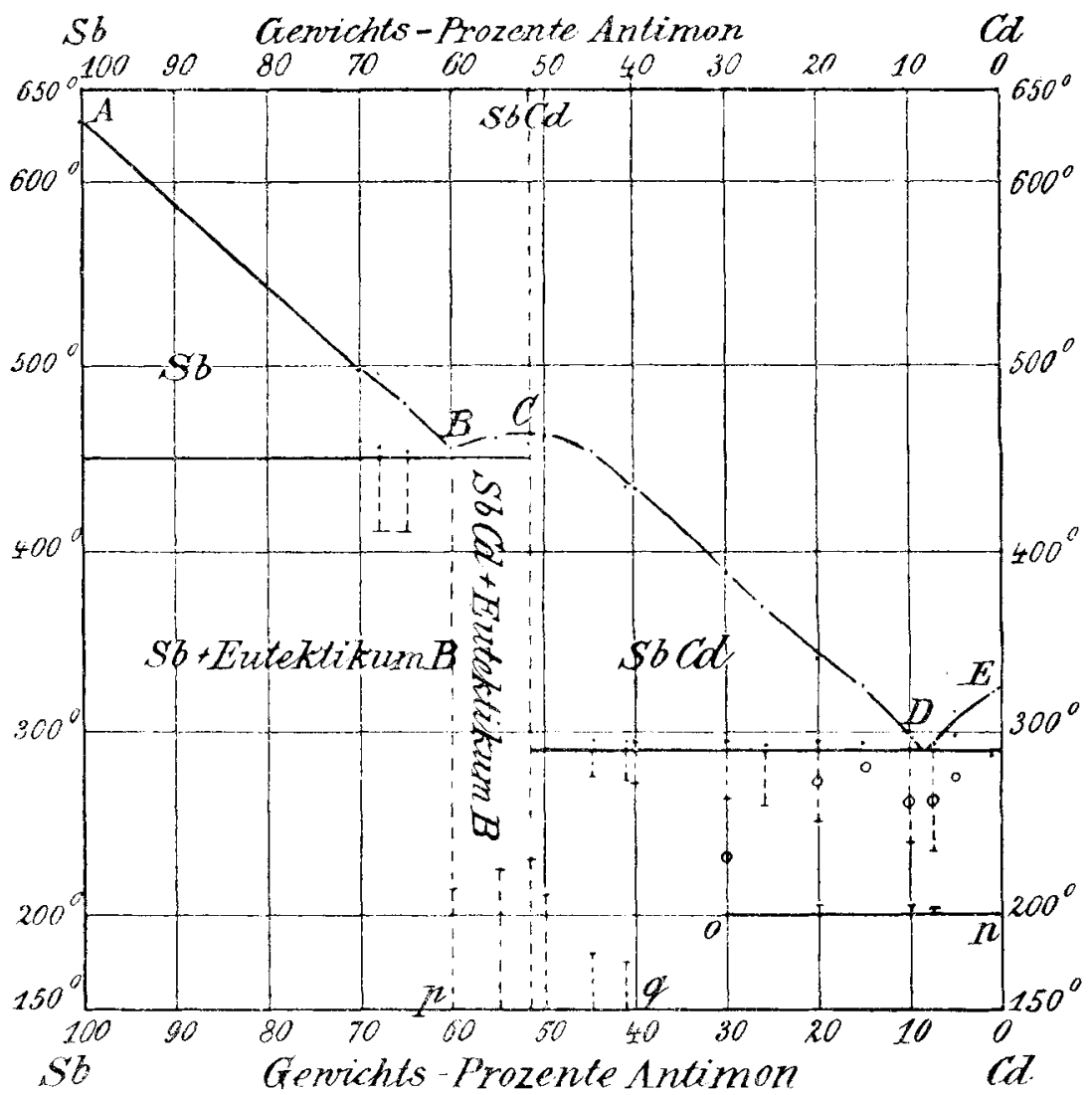

Fig. 1.

Mischkristallen der Verbindung SbCd mit Sb annehmen, doch ergab die mikroskopische Untersuchung des Regulus von der Zusammensetzung $B$ mit $60 \% \mathrm{Sb}$, dafs derselbe ausschliefslich aus einem schön lamellierten Eutektikum bestand, das sich auch im Regulus mit $55 \% \mathrm{Sb}$ und in den Reguli mit über $60 \%$ neben der Verbindung SbCd resp. freiem Antimon fand. Hieraus folgt, dafs $z$ wischen $B$ und $C$ nicht eine Reihe von Mischkristallen vorliegt, 
worauf die Abkühlungskurven hinzuweisen schienen. Der Haltepunkt der hier mafsgebenden Abkühlungskurve mit $55 \% \mathrm{Sb}$ müfste dem mikroskopischen Befunde entsprechend zwei verschiedenen Verzögerungen, der primären und der eutektischen, seinen Ursprung verdanken. Da aber die Kristallisation sich in dieser Schmelze in einem Temperaturintervall ron etwa $8^{\circ}$ vollzog, so konnten diese

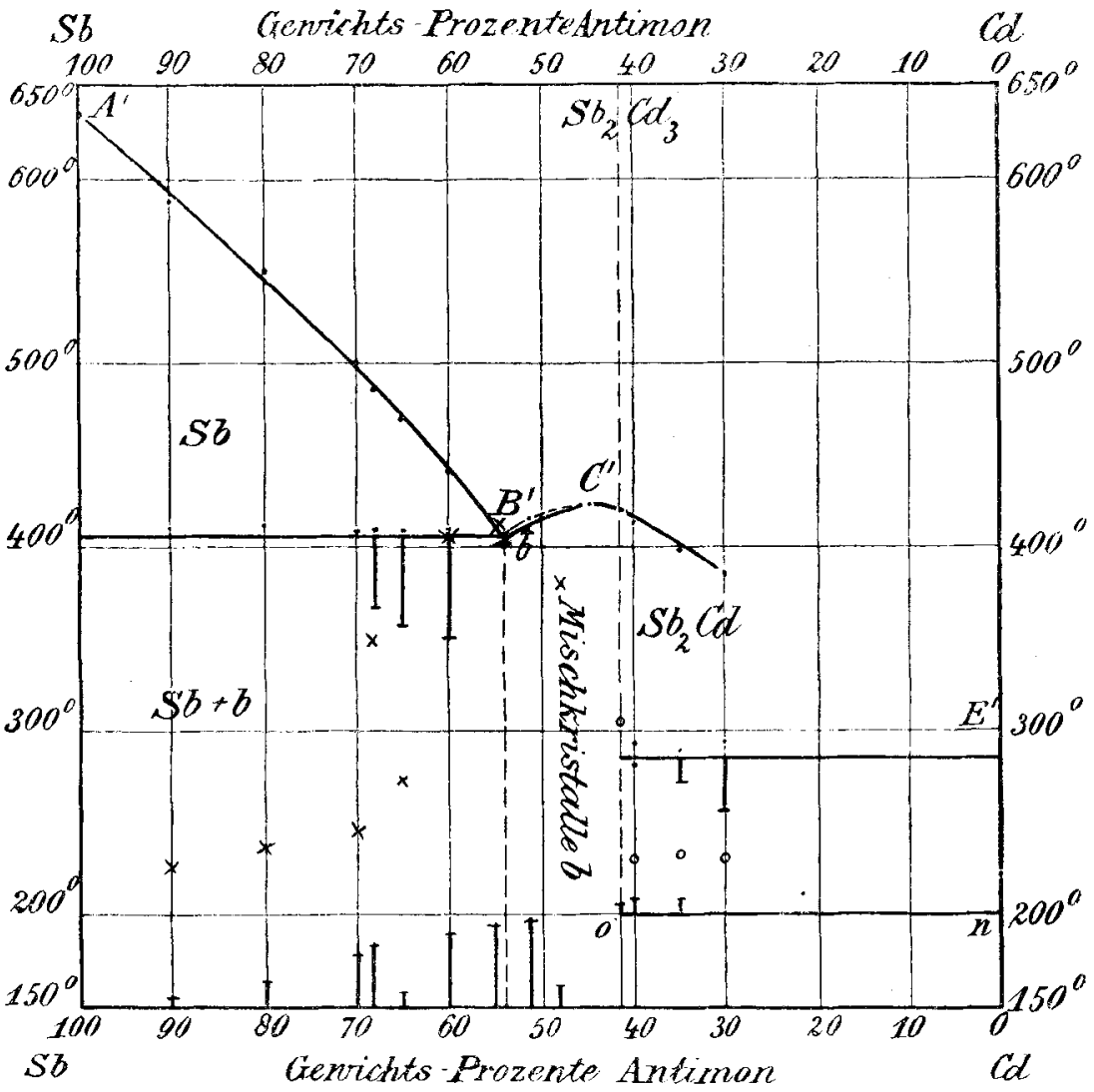

Fig. 2.

beiden Verzögerungen auf der Abkühlungskurve nicht voneinander unterschieden werden.

Die Löslichkeitskurve der Verbindung SbCd schneidet die des Antimons im eutektischen Punkte $B$ bei $60 \%$ Antimon und $455^{\circ}$. Diese eutektische Temperatur liegt um etwa $50^{\circ}$ höher als die entsprechende Temperatur, wenn an Stelle der stabilen Verbindung SbCd sich die nicht stabile Verbindung $\mathrm{Sb}_{2} \mathrm{Cd}_{3}$ ausscheidet. Schliefslich 
tinden sich auf den Abkühlungskurven von $30-5 \%$ Sb noch Haltepunkte von kleiner Zeitdauer, deren Temperatur in Fig. 1 durch Kreise kenntlich gemacht worden ist. Da die Zeitdauer dieser Haltepunkte (auf der Horizontalen on aufgetragen) bei keiner der im Diagramm vorkommenden Kristallarten ein Maximum hat, so handelt es sich hier wohl nicht um eine heteromorphe Umwandlung. Man mülste also eine chemische Reaktion zwischen Kadmium und der Verbindung SbCd annehmen, doch konnten aus der mikroskopischen Untersuchung der betreffenden Schliffe keine Indizien für eine solche Reaktion abgeleitet werden. Ein deutlicher Unterschied der langsam abgekühlten und der abgeschreckten Reguli mit 10, 7.5 and $5 \%$ Antimon konnte nicht konstatiert werden.

In Fig. 2 übersieht man das Zustandsdiagramm für den Fall, dafs aus den Schmelzen die Verbindung $\mathrm{Sb}_{2} \mathrm{Cd}_{3}$ spontan kristallisiert. Auch hier scheidet sich wiederum primär aus den Schmelzen nur eine Verbindung aus, deren Zusammensetzung wahrscheinlich mit der von Kunnakow und Konstantinow angegebenen Formel $\mathrm{Sb}_{2} \mathrm{Cd}_{3}$ übereinstimmt, denn die Zeitdauer der eutektischen Kristallisation $(292 \%$, gemessen bei 26.3 und bei $35 \% \mathrm{Sb}$, wird bei $41 \% \mathrm{Sb}$ Null. Hiermit stimmt allerdings die Lage des von mir gefundenen Maximums auf der Kurve $B^{\prime} C^{\prime}$ nicht gut überein. Das Maximum bei $C^{\prime}$ scheint, nach dem Verlauf der Schmelzkurve $B^{\prime} C^{\prime} D^{\prime}$ zu urteilen, bei etwa $45 \% \mathrm{Sb}$ zu liegen. Doch ist hierauf wohl kein besonderer Wert zu legen, da die Ausscheidung der fraglichen Verbindung fast immer mit einer Unterkühlung von $5-10^{\circ}$ eintritt. Hierdurch wird natürlich die Bestimmung der wahren Temperatur des Beginnes der Kristallisation unsicher, und man mülste, wollte man diese Frage sicher entscheiden, den Verlauf der Schmelakurve $B^{\prime} C^{\prime} D^{\prime}$ Fig. 2 unter Impfen mit Legierungen, die nur wenige Grade unter die Temperatur der Kurve $B^{\prime} C^{\prime} D^{\prime}$ abgekühlt würden, bestimmen.

Die Verbindung $\mathrm{Sb}_{2} \mathrm{Cd}_{3}$ bildet mit Antimon eine Reihe von Mischkristallen, die von $42 \%$ bis ca. $52 \% \mathrm{Sb}$ reicht. Die Gründe für die Existenz dieser Reihe von Mischkristallen sind die folgenden: Erstens sind die Abkühlungskurven der Schmelzen mit 42, 45 und $48 \% \mathrm{Sb}$ in bezug auf die Kristallisation aus der Schmelze einander ähnlich, indem sie sich untereinander und von der Abkühlungskurve der Verbindung $\mathrm{Sb}_{2} \mathrm{Cd}_{3}$ kaum unterscheiden. Hieraus ist zu schliefsen, dafs die Kurve $C^{\prime} b$, welche die Zusammensetzung der sich aus den Schmelzen ausscheidenden Mischkristalle angibt, der 
Kurve des Beginnes der Kristallisation $B^{\prime} C^{\prime}$ sehr nahe liegt. Zweitens erhält man beim Abschrecken der Reguli mit 42,48 und $52 \% \mathrm{Sb}$ von $400^{\circ}$ an in Wasser Konglomerate, die aus unter sich homogenen Kristallen bestehen. Hierbei wird die im folgenden $\mathrm{zu}$ besprechende Reaktion zwischen der Verbindung $\mathrm{Sb}_{2} \mathrm{Cd}_{3}$ und $\mathrm{Sb}$ übersprungen. Diese Reihe von Mischkristallen erstreckt sich also von der Verbindung $\mathrm{Sb}_{2} \mathrm{Cd}_{3}$ bis zum gesättigten Mischkristall, dessen Konzentration ca. $52 \%$ Antimon beträgt. Die Kristalle dieser Reihe sind bei niederen Temperaturen nicht beständig. Es tritt in denselben spontan aller Wahrscheinlichkeit nach die Bildung der Verbindung SbCd ein. Dieselbe Verbindung bildet sich in allen Reguli, welche aus Antimon und dem gesättigten Mischkristall $b$ bestehen.

Bei der Abkühlung aller Legierungen zwischen 100 und $42 \%$ Antimon tritt spontan ein erheblicher Wärmeeffekt auf, durch den die Temperatur im Maximum bis auf $50^{\circ}$ momentan ansteigt. Der Eintritt dieses Temperatursprunges, der im Diagramm für die Temperatur des Beginnes des plötzlichen Sprunges durch Kreuze angegeben ist, ist recht unregelmälsig. Man bemerkt, dals im allgemeinen dieser Temperatursprung mit steigendem Antimongehalt bei tieferer Temperatur eintritt. Die Höhe dieser Temperatursprünge, die angenähert proportional der freiwerdenden Wärmemenge und somit auch der Menge der untereinander reagierenden Kristallarten ist, ist auf der Konzentrationsachse aufgetragen. Man sieht, dafs dieselben bei etwa $53 \% \mathrm{Sb}$ ihr Maximum haben. Hieraus folgt, dafs sich bei dieser Reaktion die Verbindung SbCd bildet. Ferner trat einmal in der Legierung mit $55 \% \mathrm{Sb}$, als die Kristallisation der Verbindung $\mathrm{Sb}_{2} \mathrm{Cd}_{3}$ noch nicht beendet war, die spontane Bildung von SbCd ein, wodurch die Temperatur von der Schmelzkurve der Verbindung $\mathrm{Sb}_{2} \mathrm{Cd}_{3}$ auf die der Verbindung $\mathrm{SbCd}$ stieg. Man mufs also annehmen, dals erstens aus den Mischkristallen von 42 bis $52 \% \mathrm{Sb}$ spontan sich die Verbindung SbCd bildet, indem ein kadmiumreicheres Eutektikum hinterbleibt, und dafs zweitens in den Legierungen mit $52-100 \% \mathrm{Sb}$ aus dem gesättigten Mischkristall $b$, dessen Zusammensetzung mit derjenigen der Verbindung SbCd sehr nahe übereinstimmt, die Verbindung SbCd sich unter Austritt einer geringen Menge $\mathrm{Cd}$ bildet.

Die mikroskopische Untersuchung scheint diese Schlüsse zu bestätigen. Nach Eintritt des Temperatursprunges sieht man auf den Schlifflächen der Reguli von $100-42 \% \mathrm{Sb}$, dafs die grofsen homo- 
genen Polygone des gesättigten Mischkristalls in viele sehr erheblich kleinere Polygone, die durch ziemlich breite, tief geätzte Konturen voneinander getrennt sind, zerfallen sind. Die diesen Polygonen entsprechenden Kristalle sind offenbar die Verbindung SbCd, die uns aber hier in einer anderen Form entgegentritt als nach ihrer primären Ausscheidung aus der Schmelze. Primär aus der Schmelze ausgeschieden sind die Kristalle der Verbindung SbCd lange, makroskopisch sichtbare Säulen, nach ihrer Bildung aus den Mischkristallen mikroskopische Polyeder. Ob diese beiden Formen kristallographisch voneinander verschieden sind, lälst sich natürlich nicht entscheiden. Wir mufsten auf Grund der Abkühlungskurven annehmen, dafs die Zusammensetzung des gesättigten Mischkristalls $b$ sich von der Zusammensetzung der Verbindung nicht merklich unterscheidet. Diese Annahme wird durch die Beobachtung bestätigt, dals die Säume um die Polygone herum von $b$ aus nach $\mathrm{Sb}_{2} \mathrm{Cd}_{3}$ hin breiter werden.

\section{Zusammenfassung.}

Antimon und Kadmium bilden also zwei Verbindungen miteinander, von denen die eine, die absolut stabile, die Formel SbCd und die andere nicht stabile wahrscheinlich die Formel $\mathrm{Sb}_{2} \mathrm{Cd}_{3}$ hat. Die Verbindung $\mathrm{Sb}_{2} \mathrm{Cd}_{3}$ und die ihr zugehörige Reihe von Mischkristallen scheidet sich spontan aus allen Schmelzen von $30-100 \%$ $\mathrm{Sb}$ aus. Das Endglied dieser Reihe von Mischkristallen, der gesättigte Mischkristall $b$ mit $53 \% \mathrm{Sb}$, ist in allen spontan kristallisierten Konglomeraten von $100-53 \%$ Sb neben primär ausgeschiedenem Antimon vorhanden. Von 50-8\% Antimon wurde nie die spontane Kristallisation der Verbindung $\mathrm{Sb}_{2} \mathrm{Cd}_{3}$ beobachtet, sondern es kristallisierte immer aus diesen Schmelzen die absolut stabile Verbindung SbCd in ihren charakteristischen langen Säulen. Bei schneller Abkühlung (Abschreckung) gelingt es, diese Verbindung $\mathrm{Sb}_{2} \mathrm{Cd}_{3}$ und ihre Mischkristalle auch in den antimonreicheren Reguli mit mehr als $42 \% \mathrm{Sb}$ zu fixieren.

Impft man die Schmelzen von $100-30 \%$ Antimon mit dem Pulver derselben langsam abgekühlten Schmelze, so erhält man durchweg die langen charakteristischen Säulen der Verbindung SbCd.

Die Legierungen von $100-42 \% \mathrm{Sb}$ kann man in drei ver. schiedenen Formen darstellen. Erstens erhält man nach dem Impfen 
Konglomerate von Antimon und der Verbindung SbCd, zweitens kann man nach spontaner Kristallisation und Abschrecken von etwa $400^{\circ}$ die Mischkristalle von $\mathrm{Sb}$ und $\mathrm{Sb}_{2} \mathrm{Cd}_{3}$ resp. Konglomerate, bestehend aus dem gesättigten Mischkristall $b$ und Antimon, erhalten und drittens bekommt man nach langsamer Abküblung Konglomerate von Kristallen der Verbindung SbCd und Antimon.

Zum Schlufs sei mir gestattet, Herrn Professor T'Mmmann für seinen Rat und Beistand meinen herzlichsten Dank auszusprechen.

Göttingen, Institut für anorganische Chemie.

Bei der Redaktion eingegangen am 3. Juli 1906 